\title{
Pharyngocutaneous fistula in a Glottic carcinoma: A rare initial presentation
}

\author{
Disha Tiwari', Manjari Shah², Mansi Barthwal ${ }^{3}$, Amit Pandey ${ }^{4}$, Arun Kumar Yadav 5 , \\ Alankrita Singh ${ }^{6}$, Dev Kumar Yadav ${ }^{7}$, Madan Lal Brahma Bhatt ${ }^{8}$ \\ 1,3,4,5,6,7 Junior Resident, Department of Radiation Oncology, King George Medical University, Lucknow, Uttar Pradesh, \\ India, ${ }^{2}$ Senior Resident, Department of Radiation Oncology, Jaypee Hospital, Noida, India, ${ }^{8}$ Professor and Vice \\ Chancellor KGMU, Lucknow, Department of Radiation Oncology, King George Medical University, Lucknow, \\ Uttar Pradesh, India
}

Pharyngo-cutaneous fistula is a well-known complication following total laryngectomy in case of carcinoma larynx, however it is a very rare entity to present as an initial and sole presentation of carcinoma glottis. Here we are reporting case of glottic carcinoma presented to us with pharyngo-cutaneous fistula as an initial presentation.

Key words: Pharyngo-cutaneous fistula, Glottic carcinoma, Locally advanced laryngeal cancer
Access this article online

Website:

http://nepjol.info/index.php/AJMS

DOI: 10.3126/ajms.v8i6.18265

E-ISSN: 2091-0576

P-ISSN: 2467-9100

\section{INTRODUCTION}

Laryngeal cancer comprises approximately $1 \%$ of all malignancies and $30-50 \%$ of head and neck carcinomas ${ }^{1}$ Pharyngo-cutaneous fistula is the most common post laryngectomy complication especially after irradiation, incidence of which varies from $5 \%$ to $65 \%$ in literature. ${ }^{2,3}$ Case under reporting is an advanced glottic carcinoma with involvement of soft tissue of neck which presented as a fistulous opening as initial presentation in the midline of neck with secondary infection which is one of the rarest presentation.

\section{CASE REPORT}

A 55 year old gentle man with smoking history of around 20 years presented to radiotherapy OPD with hoarseness of voice for 7 months, intermittent throat pain for 5 months and difficulty in breathing for 1 and half months. He had a fistulous opening with discharging serosanguinous pus in the midline of neck just anterior to thyroid cartilage since 1 month as shown in Figure 1. Video-laryngoscopy of the patient revealed bilateral oedematous aryepiglottic fold, oedema of right vocal cord with bulge on left false vocal cord. Biopsy report from left vocal cord revealed well differentiated squamous cell carcinoma as shown in Figure 2. CT scan of the patient showed large hetrogenously enhancing mass in glottic, subglottic and supraglottic region with involvement of aryepiglottic fold and thyroid cartilage. It was breaching the continuity of thyroid cartilage and thyrohyoid membrane and reaching outside the strap muscle (sternothyroid, sternohyoid, omohyoid and thyrohyoid) leading to fistula formation as shown in Figure 3 and Figure 4. All haematological parameters, liver and kidney profile were within normal limit and viral markers for HIV, HBs Ag, and HCV were negative. Bacteriological culture report of the swab from the fistulous opening showed plenty of pus cells and few gram positive cocci which were identified by direct gram staining technique and pus culture revealed heavy growth of methicillin sensitive Staphylococcus aureus. Fungal evaluation of the swab was negative. Samples were taken for tubercular work up which included sputum examination and pus swab from fistula were negative for acid fast bacilli (AFB).

\section{DISCUSSION}

Extensive search of literature revealed only one reported case of carcinoma larynx who presented solely as a 


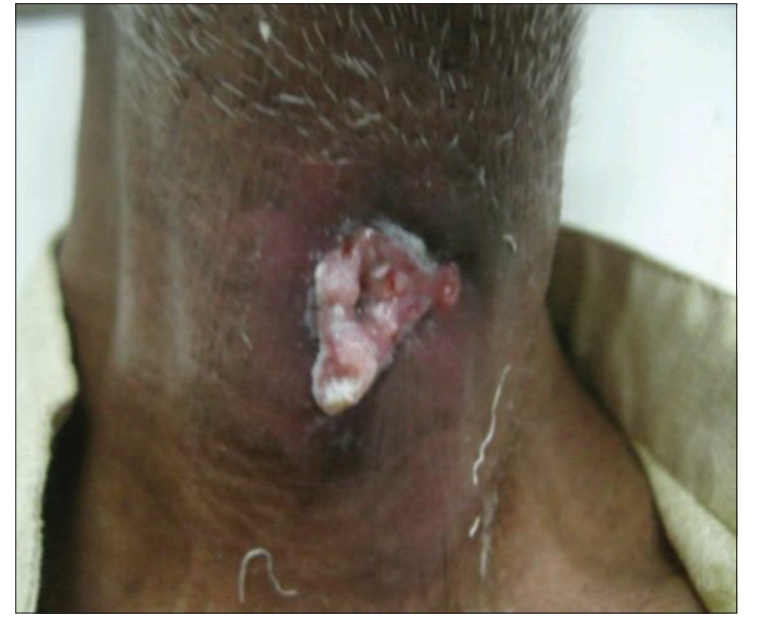

Figure 1: Fistula with discharging pus in the midline neck with surrounding erythematous changes

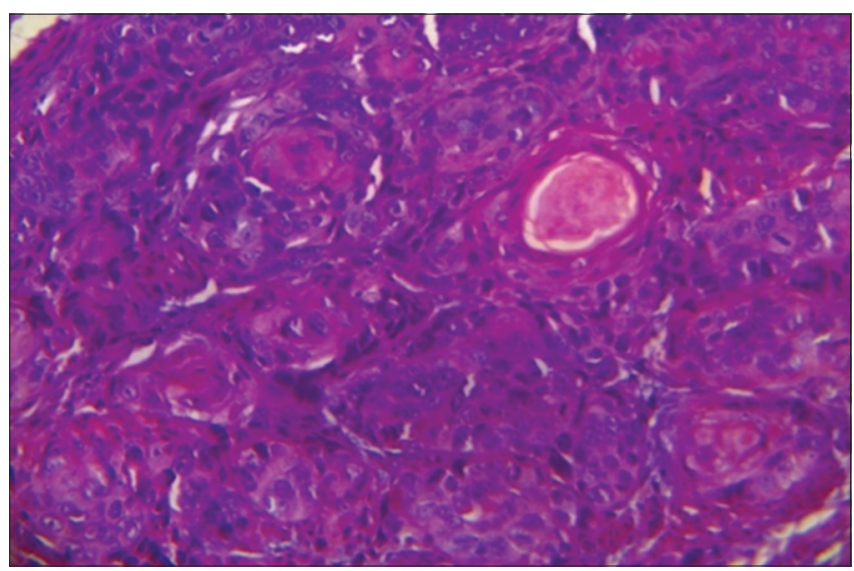

Figure 2: Histopathological image showing well differentiated carcinoma with a disorganised growth of squamous epithelium arranged in small aggregates, sheets and island of tumour infiltrating the adjacent stroma

pharyngo-cutaneous fistula in 2006 by Joe et al. ${ }^{4}$ In comparison to case reported by Joe et al which presented as fistula in neck with no other symptoms, our case presented with all features of glottic carcinoma such as hoarseness of voice, breathing difficulty along with midline fistula in the neck. Our patient with prolonged duration of disease without seeking any medical advice, progressed to form pharyngo-cutaneous fistula with no neck node and other distant metastasis clinically presented as T4aN0M0 disease. We have come across several cases of pharyngocutaneous fistula as a post operative complication of total laryngectomy but only one case of advanced carcinoma larynx which presented solely as fistula as its initial presentation. Case under reporting is another such case with one of the rarest presentation that is midline fistula of neck. Sinuses in the neck secondary to tubercular lyphadenitis also present as discharging lesions which needs to be differentiated from such type of rare presentation.

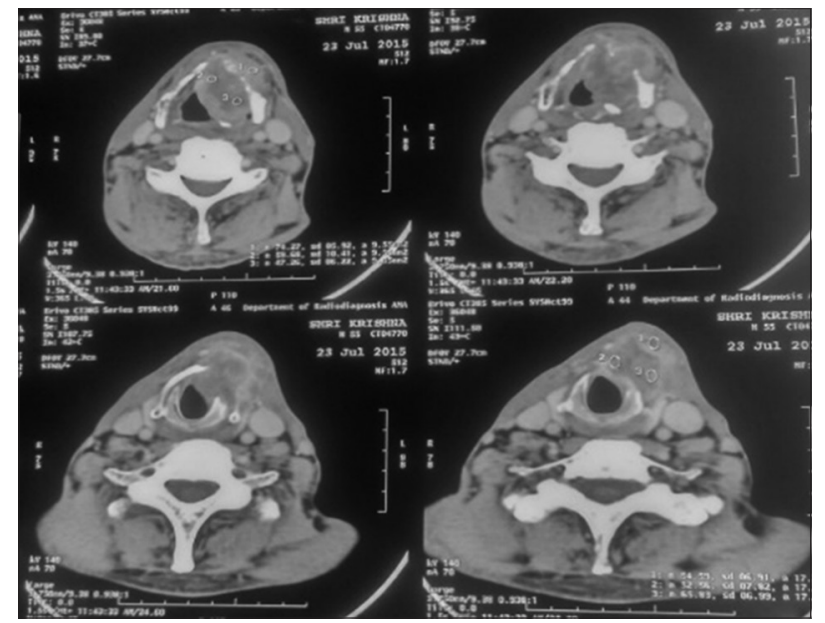

Figure 3: Axial section of contrast enhanced CT scan showing hetrogenously enhancing mass involving thyroid cartilage and soft tissue of neck reaching upto strap muscle

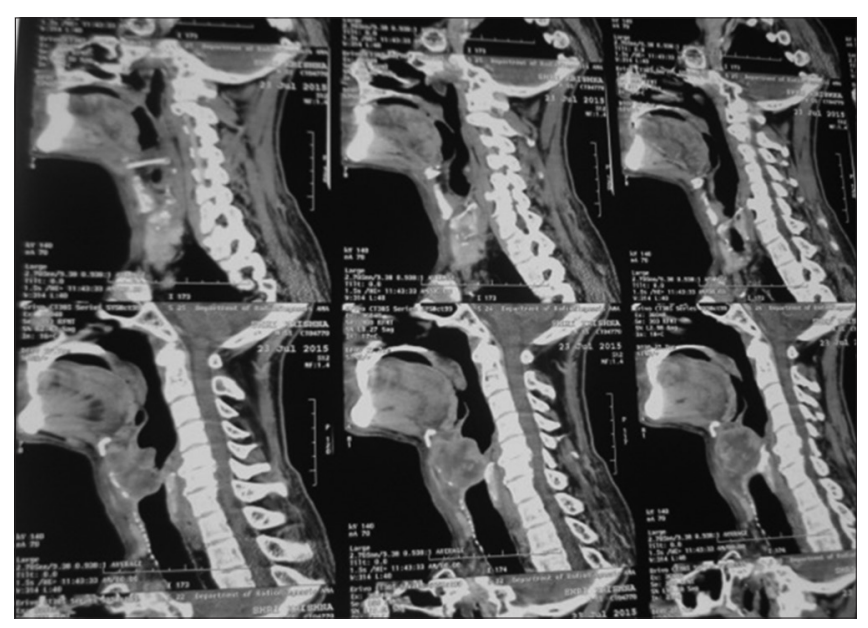

Figure 4: Sagittal section of contrast enhanced CT scan neck showing hetrogenous enhancing mass involving left thyroid cartilage and reaching the strap muscles

\section{CONCLUSION}

A midline pharyngo-cutaneous fistula presenting as an initial sign in a neglected case of glottic carcinoma is a rare presentation. However, tuberculosis which is highly prevalent in our part of world poses a dignostic dilemma. The case under discussion is a male patient in sixth decade of his life and having a long history of smoking who presented to us with a pharyngo-cutaneous fistula consequent of neglected malignancy of larynx which is a rare phenomenon. This patient is presented to clinicians with a view to have a high index of suspicion in such cases for a correct diagnosis and management.

\section{REFERENCES}

1. Landis $S$, Murray $T$, Bolden $S$ and Wingo P. Cancer statistics, 1998. CA: A Cancer Journal for Clinicians 1998;48(1):6-29. 
2. Aprigaliano $\mathrm{E}$ and Levine $\mathrm{H}$. Pharyngeal reconstruction after laryngectomy. Laryngoscope 1997; 87: 1884-1890.

3. Celikkanat $\mathrm{S}, \mathrm{Koc} \mathrm{C}$ and Ozdem C. Effect of blood transfusion on tumor recurrence and postoperative pharyngocutaneous fistula formation in patients subjected to total laryngectomy. Acta
Otolaryngol 1995; 115: 566-568.

4. Jacob J, George S, Suchit Roy BR and Rajesh PU. Malignancy larynx presenting solely as Pharyngo-cutaneous fistula. Indian Journal of Otolaryngology and Head and Neck Surgery 2006;58: (1).

\section{Authors Contribution:}

DT-Data collection, Concept of study and proof reading; MS- Contribution- Helped in preparation of first draft of manuscript; MB- Review of literature and revision of first draft of manuscript; AP- Design of the study and data collection; AKY- Contribution- Review of literature and Image collection; AS- Radiation treatment of patient and proof reading; DKY- Radiation treatment of patient and revision of manuscript; MLBB- Concept of study.

\section{Orcid ID:}

Dr Disha Tiwari: (1) http://orcid.org/0000-0003-1262-9837

Dr Manjari Shah: (D) http://orcid.org/0000-0001-9665-9624

Dr Mansi Barthwal: (1) http://orcid.org/0000-0002-9292-4571

Dr Amit Pandey: (1) http://orcid.org/0000-0002-9292-4571

Dr Arun Kumar Yadav: (1) http://orcid.org/0000-0003-2531-958X

Dr Alankrita Singh: (D) http://orcid.org/0000-0001-9932-7780

Dr Dev Kumar Yadav: (1) http://orcid.org/0000-0002-2898-0763

Dr Madan Lal Brahma Bhatt: (1) http://orcid.org/0000-0002-2898-0763

Source of Support: None, Conflict of Interest: None. 